\title{
Metapopulation Dynamics and the Evolution of Sperm Parasitism
}

\author{
K. Parvinen ${ }^{1,2}$ * \\ ${ }^{1}$ Department of Mathematics and Statistics, FI-20014 University of Turku \\ ${ }^{2}$ Evolution and Ecology Program, International Institute for Applied Systems Analysis, A-2361 \\ Laxenburg, Austria
}

\begin{abstract}
Amazon molly (Poecilia formosa) females reproduce asexually, but they need sperm to initiate the process. Such gynogenetic reproduction can be called sperm parasitism since the DNA in the sperm is not used. Since all offspring of asexually reproducing females are females, they can locally outcompete sexually reproducing ones, but their persistence is threatened by the lack of males. Therefore, the existence of Amazon mollies is puzzling. A metapopulation structure has been suggested to enable the coexistence of gynogenetic and sexual species. Previously only Levins-type metapopulation models have been used to investigate this question, but they are not defined on the individual level. Therefore we investigate the evolution of sperm parasitism in a structured metapopulation model, which incorporates both realistic local population dynamics and individual-level dispersal. If the reproduction strategy is freely evolving in a large well-mixed population or in the structured metapopulation model, strong discrimination of asexually reproducing females by males results in evolution to full sexuality, whereas mild discrimination leads to too small probability of sexual reproduction, so that the lack of males causes the extinction of the evolving population, resulting in evolutionary suicide. This classification remains the same also when both sexual reproduction and dispersal are freely evolving. Sexual and asexual behaviour can be observed at the same time in this model in the presence of a trade-off between the reproduction and dispersal traits. However, we do not observe disruptive selection resulting in the evolutionarily stable coexistence of fully sexual and fully asexual females. Instead, the presence of sexual and asexual behaviour is due to females with a mixed reproduction trait.
\end{abstract}

Keywords and phrases: Adaptive dynamics, cooperation, metapopulation

Mathematics Subject Classification: 92D15, 92D40

\section{Introduction}

Amazon molly (Poecilia formosa) is a peculiar species consisting only of females. They reproduce asexually, but their reproduction is dependent on males of other species, because sperm is needed as a developmental trigger in embryogenesis. The DNA in the sperm is not used, and therefore this kind of reproduction (gynogenesis, sperm-dependent parthenogenesis [3]) can be called sperm parasitism. The long-term presence of gynogenetic species is puzzling. They can namely grow faster in population size

*Corresponding author. E-mail: kalparvi@utu.fi 
than corresponding sexual species, because all the offspring of asexually reproducing females are females, whereas the sex ratio of sexually reproducing species is typically near one half. In the absence of other processes, such as frequency-dependent male mate preference [33], the initial increase in the population size of a gynogenetic species is expected to be followed by a population collapse due to the lack of males.

The local population dynamics [21], and metapopulation dynamics without explicit local population dynamics [22] have earlier been investigated by other researchers, who suggested that metapopulation dynamics may provide a mechanism which enables the coexistence of gynogenetic and sexual species. They investigated a Levins-type metapopulation model $[24,25]$ describing the dynamics of proportions of three types of habitat patches [22]: empty patches, habitat patch containing the sexual species alone, and habitat patch containing both the sexual and gynogenetic species. The Levins-type models are not defined on the individual level, and neglect the effect of local population sizes. In contrast, structured metapopulation models $[16,31,39]$ take local population dynamics into account. The metapopulation models in $[16,31]$ are defined in continuous time. In line with [21], in this article local population dynamics is defined in discrete time. In this article we will thus consider a model of type [39], which combines realistic local population dynamics with metapopulation-level dynamics, to investigate the evolution of sperm parasitism using the methods of adaptive dynamics $[14,15,30,32]$.

\section{Local population model}

\subsection{Local population dynamics in earlier work}

First we investigate the model by [21], who considered the following model describing the population dynamics of asexual females $A$, sexual females $F$, and males $M$ :

$$
\begin{aligned}
M_{t+1} & =f\left(N_{t}\right) m b\left(M_{t}, F_{t}, A_{t}\right) F_{t} \\
F_{t+1} & =f\left(N_{t}\right)(1-m) b\left(M_{t}, F_{t}, A_{t}\right) F_{t} \\
A_{t+1} & =f\left(N_{t}\right) b_{A}\left(M_{t}, F_{t}, A_{t}\right) A_{t}
\end{aligned}
$$

In this model, all offspring of asexual females are (asexual) females, whereas sexual females get male and (sexual) female offspring. The parameter $m$ is the sex ratio, i.e., the proportion of male offspring for a sexually reproducing female. The function $f\left(N_{t}\right)$ describes the effect of the population density $N_{t}=M_{t}+F_{t}+A_{t}$ on population growth. The functions $b$ and $b_{A}$ describe the fecundity of a sexual and an asexual female, respectively. Especially, in [21] the following fecundity functions were used

$$
b\left(M_{t}, F_{t}, A_{t}\right)=B\left(1-\exp \left(-h \frac{M_{t}}{F_{t}+(1-d) A_{t}}\right)\right)
$$

and

$$
b_{A}\left(M_{t}, F_{t}, A_{t}\right)=B\left(1-\exp \left(-h \frac{M_{t}(1-d)}{F_{t}+(1-d) A_{t}}\right)\right)
$$

The parameter $d$ denotes discrimination, i.e., the probability that a male will not mate with an asexually reproducing female.

We observe that letting $M_{t}$ to approach zero while keeping $F_{t}$ and $A_{t}$ positive, the limit fecundity is zero. This is rather natural, because if the males are extinct, there is nobody left to fertilize the females. If one, instead, keeps $M_{t}>0$, and lets both $F_{t}$ and $A_{t}$ to approach zero, the limit fecundity is equal to $B$. This observation is realistic at least for a large male population, for which the task to fertilize all females in a very small female population can be assumed to succeed. The fecundity functions in (2.2-2.3) are, however, at least mathematically problematic in low population densities. More precisely, these functions are not continuous in the origin $\left(M_{t}, F_{t}, A_{t}\right)=(0,0,0)$. The limit value namely depends on the route the limit is taken. As explained above, making first $M_{t}$ to approach zero and only then letting $F_{t}$ and $A_{t}$ approach zero, we obtain the value 0 , which is different from $B$, which is obtained if first $F_{t}$ and $A_{t}$ approach zero and only then $M_{t}$ approaches zero. In addition to causing mathematical problems, such a 
situation is even not realistic. When all population densities approach zero, it will become increasingly difficult to the individuals to find a mating partner. Therefore, the limit value of fecundity in the origin should be zero. For this reason, we will not use the fecundity functions (2.2-2.3), but instead take a mechanistic approach $[5,9-11,49]$, and derive fecundity functions from first principles. Such an approach is important also from the evolutionary perspective [46].

One might argue, that adding one male into a population consisting of only females will have a dramatic effect on the fecundity of the females. When making such an argument, one at the same time assumes a different modelling approach. Here we have used deterministic models with population densities, in which adding one individual has no practical effect on the population density. If one single individual is allowed to have such an effect, it is necessary to consider either population models with a small (finite) total number of individuals, or metapopulation models, in which at least the local population is small (finite). In both cases, demographic stochasticity must be taken into account. Furthermore, in such a case the arguments of the function describing the average fecundity of a female are natural numbers (numbers of different individuals). Especially the number of females must be at least one, and thus it is not meaningful (and also not necessary) to ask what would be the limit of such a function in the origin. We conclude that an infinitesimally small change in population densities cannot be assumed to have a dramatic effect on fecundity.

\subsection{Deriving fecundity functions from first principles}

We assume that matings between males and females occur during a specific mating season, the time within which is denoted by $\tau, 0 \leqslant \tau \leqslant 1$. Let $\hat{M}$ denote the population density of sperm-rich males, and $\hat{F}$ the population density of unfertilized females during the mating season. Initially all males are sperm rich, so that $\hat{M}(0)=\hat{M}_{0}=M_{t}$. For simplicity, we assume that discrimination occurs first, so that $\hat{F}(0)=\hat{F}_{0}=F_{t}+(1-d) A_{t}$.

In a well-mixed population matings occur according to the law of mass action, with the rate $\delta$. The parameter $\xi$ describes the proportion of such meetings which result in the male becoming exhausted of sperm. The parameter $\gamma$ describes the proportion of such meetings which result in the female becoming fertilized. We thus obtain

$$
\begin{aligned}
\frac{d}{d \tau} \hat{M}(\tau) & =-\delta \xi \hat{M}(\tau) \hat{F}(\tau) \\
\frac{d}{d \tau} \hat{F}(\tau) & =-\delta \gamma \hat{M}(\tau) \hat{F}(\tau)
\end{aligned}
$$

After solving the differential equations (2.4), we obtain the fecundity function

$$
b\left(\hat{M}_{0}, \hat{F}_{0}\right):=B \frac{\hat{F}_{0}-\hat{F}(1)}{\hat{F}_{0}}= \begin{cases}B \gamma \hat{M}_{0} \frac{e^{\delta \xi \hat{F}_{0}}-e^{\delta \gamma \hat{M}_{0}}}{\xi \hat{F}_{0} \delta \xi \hat{F}_{0}-\gamma \hat{M}_{0} e^{\delta \gamma \hat{M}_{0}}}, & \text { for } \gamma \hat{M}_{0} \neq \xi \hat{F}_{0} \\ B \gamma \hat{M}_{0} \frac{\delta}{1+\delta \gamma \hat{M}_{0}}, & \text { for } \gamma \hat{M}_{0}=\xi \hat{F}_{0}\end{cases}
$$

\subsection{Generalized local population dynamics}

As mentioned earlier, for females that are able to reproduce through gynogenesis, males act like a public resource. Therefore, gynogenesis can be seen as sperm parasitism. In order to study the evolution of such behaviour, we make the following generalization to the model of [21]: We assume that the reproduction behaviour of a female is determined by the inherited (from the mother) trait $s$, which is the probability that the female will attempt to reproduce sexually (the corresponding probability for asexual reproduction is $1-s)$. Note that the population is assumed to be semelparous: the population in the next time-step consists only of offspring of the present population, because adults are assumed to die after reproduction. This means that each female will reproduce at most once. Let $F^{i}$ denote the population density of females with reproduction strategy $s_{i}, i=1, \ldots, n$. 
A sexually reproducing female has fecundity $b\left(F_{t}, M_{t}\right)$, but only the proportion $(1-m)$ of its offspring are female. An asexually reproducing female may be discriminated, and thus has smaller average fecundity $(1-d) b\left(F_{t}, M_{t}\right)$, but all its offspring are female. Now the local population dynamics becomes

$$
\begin{aligned}
F_{t+1}^{i} & =f\left(N_{t}\right) b\left(\hat{F}_{t}, M_{t}\right)\left[\left(1-s_{i}\right)(1-d)+s_{i}(1-m)\right] F_{t}^{i} \\
M_{t+1} & =f\left(N_{t}\right) b\left(\hat{F}_{t}, M_{t}\right) \sum_{i=1}^{n} s_{i} m F_{t}^{i},
\end{aligned}
$$

where $M_{t}$ is the male population density,

$$
N_{t}=M_{t}+\sum_{i=1}^{n} F_{t}^{i}
$$

is the total population density, and

$$
\hat{F}_{t}=\sum_{i=1}^{n}\left[\left(1-s_{i}\right)(1-d)+s_{i}\right] F_{t}^{i}
$$

is the population density of females that are not discriminated.

In numerical examples, we have assumed that the effect of the total population density on population growth is

$$
f(N)=\frac{1}{(1+\alpha N)^{\beta}} .
$$

Figure 1 illustrates equilibrium population densities with respect to the reproduction strategy $s$. For the sex ratio $\frac{F}{M}$ at a monomorphic equilibrium we obtain the analytical expression (See also Figure 1c)

$$
\frac{F}{M}=\frac{(1-s)(1-d)+s(1-m)}{s m}
$$

a)

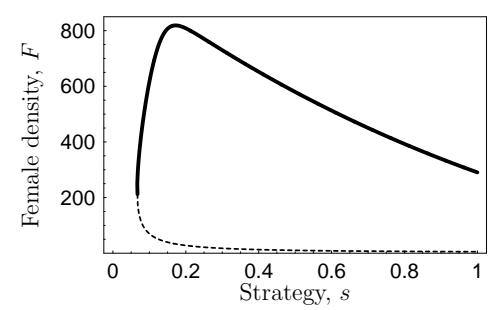

b)

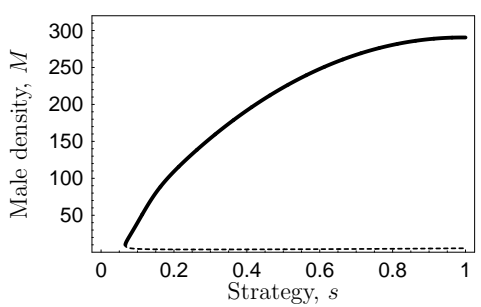

c)

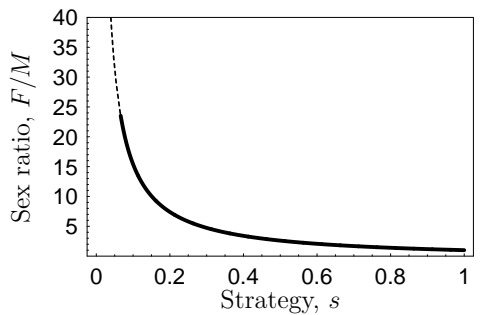

FiguRE 1. Female (a) and male (b) population densities at equilibrium of (2.6) with respect to the reproduction strategy $s$. The thick curve corresponds to a stable equilibrium, and the dashed curve to an unstable equilibrium. (c) The theoretical sex ratio $\frac{F}{M}$ at equilibrium (2.10), thin dashed curve, coincides with the numerically obtained one from panels a and b, thick curve, for those values of the strategy $s$ that a stable positive equilibrium exists. Parameters: $d=0.2, m=0.5, f(N)$ as in (2.9) with $\alpha=0.001$, $\beta=2$, fecundity function $b$ as in (2.5) with $B=5, \delta=0.1, \gamma=1, \xi=0.1$. 


\section{Evolution of sperm parasitism}

\subsection{Adaptive dynamics}

Invasion fitness [32] is a central concept in adaptive dynamics. It is defined as the long-term exponential growth rate $r\left(s_{\text {mut }}, E_{\text {res }}\right)$ of a rare mutant $s_{\text {mut }}$ in an environment $E_{\text {res }}$ set by the resident. If the invasion fitness of a mutant is positive, it is able to grow in population size. Therefore it may invade and become a new resident itself. Random small mutations are assumed to happen rarely, so that the population dynamics has settled to an attractor before the next mutation happens. These mutation-invasion events result in the change of the strategy of the individuals constituting the population. These events define a trait-substitution sequence. Each element of such a sequence is either a strategy or a set of several strategies, replacing the phenotype(s) that were previously present. Although each trait-substitution sequence is a realization of a stochastic process, there are analytical methods of adaptive dynamics which tell what will happen to all possible trait-substitution sequences in the long run.

If the invasion fitness of any mutant is non-positive in the environment set by a specific resident, then this resident strategy is uninvadable, and called as an evolutionarily stable strategy, or ESS [27]. In this case the resident strategy is necessarily a (local) fitness maximum and thus the selection gradient, i.e., the derivative of invasion fitness with respect to the strategy of the mutant, is zero at such points,

$$
\left.\frac{\partial}{\partial s_{\mathrm{mut}}} r\left(s_{\mathrm{mut}}, E_{\mathrm{res}}\right)\right|_{s_{\mathrm{mut}}=s_{\mathrm{res}}}=0
$$

and the second derivative $\left.\frac{\partial^{2}}{\partial s_{\mathrm{mut}}^{2}} r\left(s_{\mathrm{mut}}, E_{\mathrm{res}}\right)\right|_{s_{\mathrm{mut}}=s_{\mathrm{res}}}<0$ is negative. Strategies for which the selection gradient is zero, are called evolutionarily singular strategies [14]. Such a singular strategy $s^{*}$ is convergence stable or an evolutionary attractor if the repeated invasion of nearby mutant strategies into resident strategies will lead to the convergence of resident strategies towards $s^{*}$ [6]. If an evolutionary attractor is also evolutionarily stable, it is called a continuously stable strategy (CSS; 8) and it is a feasible final outcome of an evolutionary process. In case the second derivative $\left.\frac{\partial^{2}}{\partial s_{\text {mut }}^{2}} r\left(s_{\text {mut }}, E_{\text {res }}\right)\right|_{s_{\text {mut }}=s_{\text {res }}}>0$ is positive for a monomorphically attracting singular strategy, this singular strategy is not unbeatable, and evolutionary branching [14] may occur because of disruptive selection. This phenomenon can result in the evolutionary emergence of cooperators and defectors in an initially monomorphic population $[4,7]$.

We can illustrate the monomorphic adaptive dynamics by drawing pairwise invasibility plots (PIP) [26, 51], where the sign of the invasion fitness is displayed in dependence on resident and mutant strategies (Figures 2 and 4). Because the resident population is at a population-dynamical equilibrium, a mutant population with the same strategy as the resident will have a neutral growth rate. Therefore there is a zero-contour at the diagonal $s_{\text {mut }}=s_{\text {res }}$ in pairwise invasibility plots. Evolutionarily singular strategies lie at those points where other zero-contours cross the diagonal.

\subsection{Evolution of sperm parasitism in a well-mixed population}

Now assume that the resident population has reached a population-dynamical attractor, in which $N_{t}$ is the total population density, $M_{t}$ is the male population density, and $\hat{F}_{t}$ is the population density of undiscriminated females. Analogous to (2.6), a rare mutant population with strategy $s_{\text {mut }}$ will initially grow according to

$$
F_{t+1}^{\text {mut }}=f\left(N_{t}\right) b\left(\hat{F}_{t}, M_{t}\right)\left[\left(1-s_{\text {mut }}\right)(1-d)+s_{\text {mut }}(1-m)\right] F_{t}^{\text {mut }} .
$$

Note that the population densities $N_{t}, M_{t}$, and $\hat{F}_{t}$ are determined only by the resident species according to equations (2.6-2.8). The mutant population is at least initially so small, that it does not affect the resident populations, or the environment they determine. Therefore, the (discrete-time) invasion fitness 
of a mutant in such an environment is

$$
\begin{aligned}
R(s_{\mathrm{mut}}, \underbrace{\mathbf{N}, \mathbf{M}, \hat{\mathbf{F}}}_{E_{\mathrm{res}}}) & =\lim _{T \rightarrow \infty} \sqrt[T]{\prod_{t=1}^{T} f\left(N_{t}\right) b\left(\hat{F}_{t}, M_{t}\right)\left(\left(1-s_{\mathrm{mut}}\right)(1-d)+s_{\mathrm{mut}}(1-m)\right)} \\
& =\left(\left(1-s_{\mathrm{mut}}\right)(1-d)+s_{\mathrm{mut}}(1-m)\right) \lim _{T \rightarrow \infty} \sqrt[T]{\prod_{t=1}^{T} f\left(N_{t}\right) b\left(\hat{F}_{t}, M_{t}\right) .}
\end{aligned}
$$

Because the resident population is on an attractor, the discrete-time invasion fitness of any resident strategy $s_{\text {res }}$ must be equal to one. Therefore

$$
R\left(s_{\mathrm{mut}}, E_{\mathrm{res}}\right)=\frac{\left(1-s_{\mathrm{mut}}\right)(1-d)+s_{\mathrm{mut}}(1-m)}{\left(1-s_{\mathrm{res}}\right)(1-d)+s_{\mathrm{res}}(1-m)}=\frac{1-d+s_{\mathrm{mut}}(d-m)}{1-d+s_{\mathrm{res}}(d-m)} .
$$

Evolutionary analysis using the invasion fitness (3.4) is straightforward. Because the invasion fitness is linear with respect to $s_{\text {mut }}$, the derivative

$$
\frac{\partial}{\partial s_{\mathrm{mut}}} R\left(s_{\mathrm{mut}}, E_{\mathrm{res}}\right)=\frac{d-m}{\left(1-s_{\mathrm{res}}\right)(1-d)+s_{\mathrm{res}}(1-m)}
$$

does not depend on $s_{\text {mut }}$. Especially, the fitness gradient is

$$
\left.\frac{\partial}{\partial s_{\mathrm{mut}}} R\left(s_{\mathrm{mut}}, E_{\mathrm{res}}\right)\right|_{s_{\mathrm{mut}}=s_{\mathrm{res}}}=\frac{d-m}{\left(1-s_{\mathrm{res}}\right)(1-d)+s_{\mathrm{res}}(1-m)} .
$$

In case there is strong discrimination, $d>m$, the fitness gradient is always positive, and the reproduction strategy $s$ is expected to evolve to $s=1$, in which case all reproduction is sexual (Figure 2a).

In case of light discrimination, $d<m$, the fitness gradient is always negative. Therefore, evolution favours reducing $s$ (Figure 2b). However, when $s$ is too low, the population is not viable (Figure 1), because the lack of males causes too low probability of successful mating. Nevertheless, strategies with lower $s$ than that of the resident have positive fitness, and can at least initially increase in population size. The strategy of the population is thus expected to decrease and to approach a boundary of viability. Eventually, an unviable strategy is able to invade the resident population, but will at the same time cause the extinction of the evolving population, resulting in evolutionary suicide $[12,17,18,38,42,43]$.

\subsection{Metapopulation dynamics and equilibria}

We have already observed that a fully asexual gynogenetic species and a related sexual species are not likely to coexist in a well-mixed population. If the two species occupy otherwise the same ecological niche, and discrimination is not too strong, the asexually reproducing individuals always have reproductive advantage over the sexually reproducing individuals, which will eventually lead to the collapse of the population due to the lack of males. Henceforth, spatial models may provide an explanation for such coexistence [22]. In specific, a metapopulation is a collection of local populations living in habitat patches connected with dispersal. Richard Levins [24,25] presented the first metapopulation model, which described the dynamics of proportions of occupied and empty habitat patches. A Levins-type metapopulation describing the situation could consist of local populations with either the sexual species alone, or both the sexual and the asexual one (gynogenetically reproducing all-female species) together. As the matter of fact, it has been demonstrated [22], that in such a model coexistence is possible, provided that the corresponding patch colonization rates are suitably chosen. However, such patch colonization rates are not individual-level properties. In reality the colonization of a patch requires individuals (or seeds) to disperse into this patch from another patch. Furthermore, after such an dispersal event, the local population in this patch will at least initially be small, and it takes time for the local population size to 
a) $d=0.7$

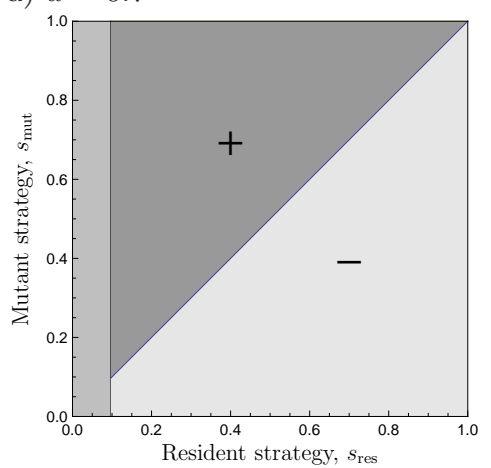

b) $d=0.2$

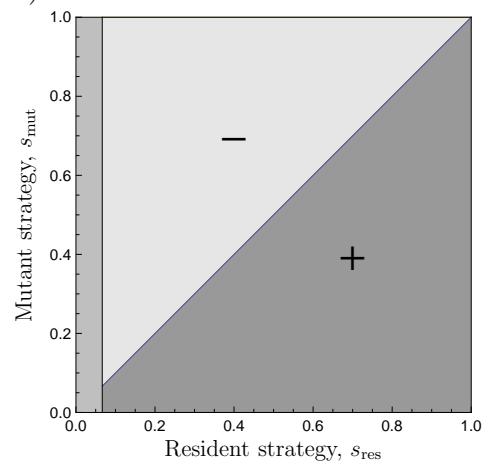

FIgURE 2. Pairwise invasibility plots for different values of the discrimination parameter $d$. (a) When $d>m$, the reproduction strategy $s$ evolves to the maximum value 1 . (b) When $d<m$, lower $s$ are always favoured by natural selection. Too low strategies are, however, not viable, and therefore evolutionary suicide occurs. Parameters: a) $d=0.7$, b) $d=0.2$. Other parameters (as in Figure 1): $B=5, \delta=0.1, \gamma=1, \xi=0.1, \alpha=0.001$, $\beta=2, m=0.5$

grow to such a size, that this local population can be expected to produce dispersers to other, potentially empty, patches. Therefore, for more thorough investigation, one should not neglect the effect of local population dynamics. To study the evolution of sperm parasitism in such structured metapopulation models is one aim of this article.

There are many articles in the literature, which investigate various evolutionary questions in structured metapopulation models [16, 18,31,39, 40,44,45]. Especially, [39] presented a general structured metapopulation model, in which local population dynamics is defined in discrete time. We will use that model as a basis for our model construction. The landscape is assumed to consist of an infinite number of habitat patches. Patches differ in the size and composition of the local populations, but are otherwise equal. In each season, first reproduction happens according to (2.6). After that, the fraction $e$ of the offspring disperses and enters the disperser pool.

The newly emigrated offspring are not yet able to immigrate into the patches. They will survive to the next season in the disperser pool with probability $\pi$, otherwise they die. All other dispersers in the disperser pool will immigrate into a habitat patch. Immigrants choose their patch at random, independently of the local population size. Therefore, the amount of immigrants $I_{t}$ each patch will receive is equal to the disperser pool size. We could alternatively assume that dispersers could stay in the dispersal pool for longer time. However, it has been shown [39] that metapopulation dynamical equilibria and invasion fitness depend on the events in the dispersal pool only through the probability to survive dispersal, which in this case is equal to $\pi$.

Random catastrophes occurring with probability $\mu$ can occasionally kill all individuals in the patch, thus setting the local population size to zero. Such a patch remains habitable, and can be re-colonized by dispersers from the disperser pool. If a catastrophe has not happened, the local population densities in a patch will change according to

$$
\begin{aligned}
F_{t+1}^{i} & =\left(1-e_{F, i}\right) f\left(N_{t}\right) b\left(\hat{F}_{t}, M_{t}\right)\left[\left(1-s_{i}\right)(1-d)+s_{i}(1-m)\right] F_{t}^{i}+I_{t}^{i} \\
M_{t+1} & =\left(1-e_{M}\right) f\left(N_{t}\right) b\left(\hat{F}_{t}, M_{t}\right) \sum_{i=1}^{n} s_{i} m F_{t}^{i}+I_{t}^{M},
\end{aligned}
$$


where $I_{t}^{i}$ and $I_{t}^{M}$ describe the amounts of female and male immigrants each patch will receive. These immigrants consist of all those emigrants who emigrated at time $t-1$ and survived.

$$
\begin{aligned}
I_{t}^{i} & =\pi \int e_{F, i} f(N) b(\hat{F}, M)\left[\left(1-s_{i}\right)(1-d)+s_{i}(1-m)\right] F^{i} n_{t-1}\left(d M, d F^{1}, \ldots, d F^{n}\right) \\
I_{t}^{M} & =\pi \int e_{M} f(N) b(F, M) \sum_{i=1}^{n} s_{i} m F^{i} n_{t-1}\left(d M, d F^{1}, \ldots, d F^{n}\right),
\end{aligned}
$$

where $n_{t-1}\left(M, F^{1}, \ldots, F^{n}\right)$ is the size distribution of the metapopulation at time $t-1$. The quantity $\int_{A} n_{t-1}\left(d M, d F^{1}, \ldots, d F^{n}\right)$ is thus the probability that the local population sizes in a patch belong to the set $A \subset \mathbb{R}_{+}^{n+1}$ at time $t-1$.

Although the dynamics of $n_{t}\left(M, F^{1}, \ldots, F^{n}\right)$ follow from straightforward book-keeping of local population dynamics, we do not need to express it in more detail, because for finding metapopulation-dynamical equilibria we obtain an easier expression. In a metapopulation-dynamical equilibrium, the amounts of immigrants $I_{t}^{i}$ and $I_{t}^{M}$ and the population size distribution $n_{t}\left(M, F^{1}, \ldots, F^{n}\right)$ are constant. This distribution has a special form [39], because all patches of age $\tau$ (time since the last catastrophe happened in a patch) have the same population sizes $M_{\tau}, F_{\tau}^{1}, \ldots, F_{\tau}^{n}$, which can be solved from (3.7) with the initial condition $M_{0}=F_{0}^{1}=\cdots=F_{0}^{n}=0$ using constant values of $I^{i}$ and $I^{M}$. Finally, the correct values of $I^{i}$ and $I^{M}$ need to satisfy the following conditions from which they can be solved at least numerically:

$$
\begin{aligned}
I^{i} & =\pi \sum_{\tau=0}^{\infty} e_{F, i} f\left(N_{\tau}\right) b\left(\hat{F}_{\tau}, M_{\tau}\right)\left[\left(1-s_{i}\right)(1-d)+s_{i}(1-m)\right] F_{\tau}^{i} v(\tau) \\
I^{M} & =\pi \sum_{\tau=0}^{\infty} e_{M} f\left(N_{\tau}\right) b\left(F_{\tau}, M_{\tau}\right) v(\tau) \sum_{i=1}^{n} s_{i} m F_{\tau}^{i},
\end{aligned}
$$

where $v(\tau)=\mu(1-\mu)^{\tau}, \tau=0, \ldots, \infty$ is the age distribution of patches at equilibrium.

\subsection{Invasion fitness and the metapopulation reproduction ratio}

The invasion fitness [32] is defined as the long-term exponential growth rate $r\left(s_{\text {mut }}, E_{\text {res }}\right)$ of a rare mutant $s_{\text {mut }}$ in an environment $E_{\text {res }}$ set by the resident. The direct computation of invasion fitness is complicated in structured metapopulation models. For this reason, [16] and [31] presented the metapopulation reproduction ratio

$$
R_{m}\left(s_{\text {mut }}, E_{\text {res }}\right) \text {. }
$$

Consider a single mutant disperser arriving in a patch. This mutant may reproduce and thus gain descendants in this patch, but this will not necessarily happen because of demographic stochasticity. As long as this mutant or at least one of its descendants is present in the local population, we call it a mutant colony. Again, because of demographic stochasticity and catastrophes, the mutant colony will eventually go extinct. During its lifetime, some mutants will emigrate from this mutant colony to the disperser pool. Their average number is the metapopulation reproduction ratio $R_{m}$ of the mutant. In other words, it is the expected number of successful dispersers produced by a typical mutant colony initiated by a single mutant disperser. The models in [16] and [31] were defined in continuous time. The quantity $R_{m}$ can analogously be calculated in a discrete-time metapopulation model [39]. The definition of $R_{m}$ using the notation of this article is presented in detail in the Appendix. The invasion condition $r\left(s_{\text {mut }}, E_{\text {res }}\right)>0$ is equivalent to $R_{m}\left(s_{\text {mut }}, E_{\text {res }}\right)>1$, and thus $R_{m}$ is a proxy for invasion fitness, and can be used to study evolution in metapopulation models.

\subsection{Evolution of sperm parasitism in a metapopulation}

We next investigate the evolution of the reproduction strategy $s$ in the metapopulation model described above. Our numerical results show that evolutionary dynamics are analogous to the well-mixed case: with 
strong discrimination $(d>m)$ the reproduction strategy $s$ evolves to $s=1$, and with light discrimination $(d<m)$ evolution favours reducing $s$, which will lead to evolutionary suicide. We can also prove this result analytically using methods analogous to the well-mixed case (not shown). This result contrasts with results in the Levins-type model [22], in which the coexistence of full asexual species and a full sexual species is possible, provided that the corresponding patch colonization rates are suitably chosen. We, however, need to note that colonization rates in a Levins-type metapopulation model are not properties of individuals. In structured metapopulation models instead, we describe how individual behaviour affects reproduction and dispersal, which in turn determine how populations with different reproduction strategies will grow. In order to two different strategies to coexist, both should have an ecological niche, which they can exploit better than the other strategy. So far we have assumed that individuals differ only in their reproduction behaviour, and depending on the relative magnitude of the parameters $d$ and $m$, either the smaller strategy always performs better than the larger one, or vice versa. Thus, in order to observe coexistence of two (or more) strategies, individuals need to differ also in other properties than their reproduction behaviour.

Next we let also the dispersal probability of females to freely evolve due to natural selection. The strategy of an individual is then the vector $\left(s, e_{F}\right)$. For vector-valued strategies, pairwise invasibility plots are not fully sufficient to illustrate the evolutionary dynamics. Instead, Figure 3 illustrates the expected direction of evolution, given by the two-dimensional fitness gradient, by showing its isoclines. From Figure 3 we observe that this situation still leads to either full sexuality $(s=1)$ or too small $s$ resulting in evolutionary suicide.
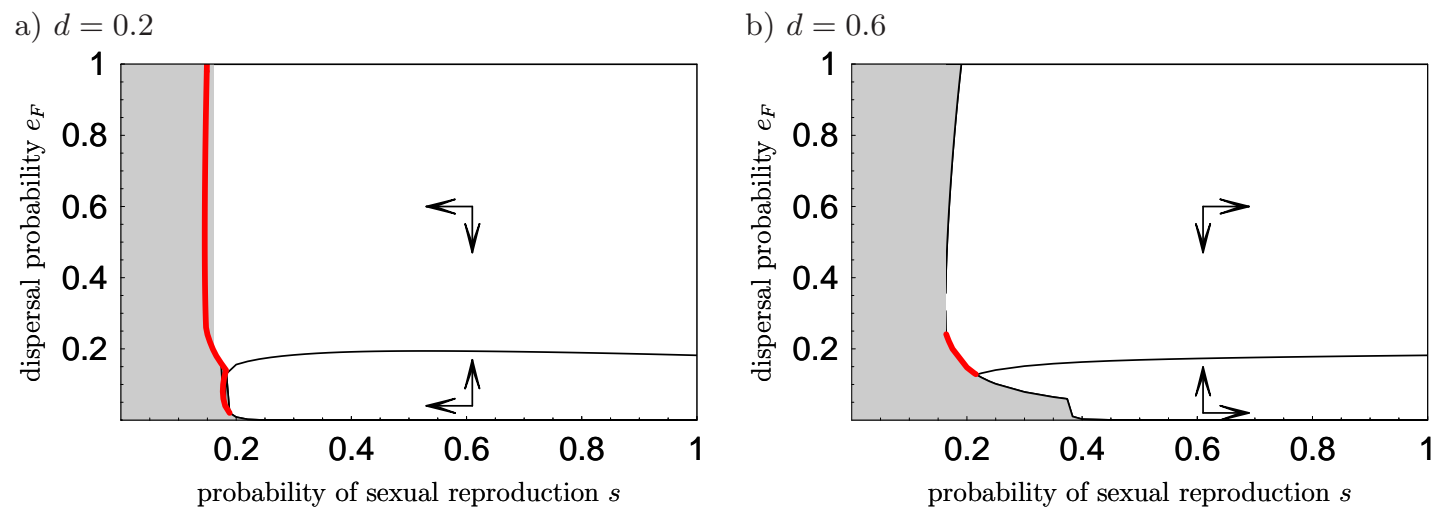

FIgURE 3. Isoclines of the two-dimensional fitness gradient, when both the reproduction strategy $s$ and the dispersal probability $e_{F}$ of females are freely evolving. Arrows show the direction of the fitness gradient. Metapopulation parameters: $\mu=0.05, m_{M}=$ $0.2, \pi=0.85$, other parameters: $\alpha=0.001, \beta=2, B=10, m=0.5, \delta=0.1, \gamma=0.5$, $\xi=0.1$.

Next we study what happens if the reproduction strategy $s$ and the female dispersal probability $e_{F}$ are traded off, so that $e_{F}=z(s)$. For simplicity, here we only consider linear trade-offs, in which

$$
e_{F}=T\left(c_{1}+c_{2}(s-0.5)\right) \text {. }
$$

The function $T$ is included to avoid meaningless probabilities, so that

$$
T(x)= \begin{cases}0, & \text { for } x<0 \\ x, & \text { for } 0 \leqslant x \leqslant 1 \\ 1, & \text { for } x>1\end{cases}
$$


We do not claim that the dispersal probability and the reproduction strategy would normally be coupled with a one to one correspondence. Our aim is to investigate whether such dependence would be sufficient for observing coexistence of sexual and asexual species, that are otherwise ecologically equal. Figure 4 illustrates that in the presence of a positive trade-off between the probability of sexual reproduction and the dispersal probability, intermediate singular strategies may appear. More precisely, when the trade-off multiplier is not too large $\left(c_{2}=0.2\right.$ in Figure 4$)$, for low discrimination probabilities $d$, there is selection for too low $s$ so that evolutionary suicide happens (Figure 4a). When $d$ is increased, instead of a sudden change to evolution to full sexuality, an intermediate evolutionarily singular strategy appears, which is also evolutionarily stable (Figure 4bc). Evolution to full sexuality does occur when $d$ is sufficiently increased (Figure 4d). This evolutionary bifurcation is illustrated also in Figure 5a.

a) $d=0.4$

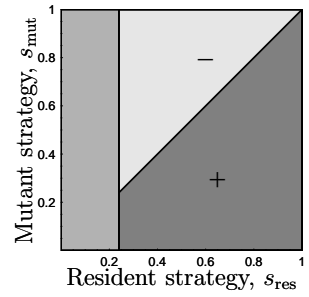

b) $d=0.47$

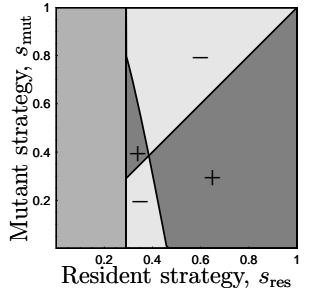

c) $d=0.495$

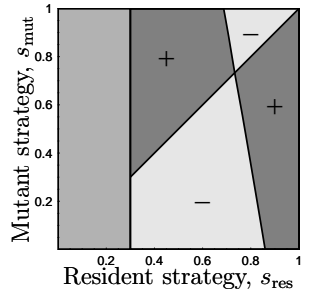

d) $d=0.505$

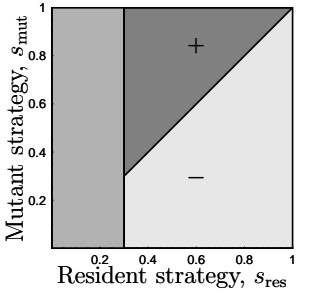

FiguRE 4. Pairwise invasibility plots with respect to the reproduction strategy $s$, when $e_{F}=0.1+c_{2}(s-0.5)$ with $c_{2}=0.2$. Other parameters as in Figure 3a, including $m=0.5$.

As noted above, without the trade-off in dispersal, in case $d<m$, evolution favours decreasing $s$ until the extinction boundary is reached, so that evolutionary suicide happens. When a trade-off is incorporated, the lower boundary of viability becomes evolutionarily repelling for $c_{2}$ large enough (Figure $5 \mathrm{bc}$ ), and an intermediate evolutionarily singular strategy appears. On the other hand, increasing $c_{2}$ when $d>m$ can cause the strategy boundary of full sexualily $(s=1)$ to become evolutionarily repelling (Figure 5bc), so that evolution again reaches an intermediate evolutionarily singular strategy. Figure 5c illustrates that the presence of an intermediate evolutionarily singular strategy occurs with a wide range of parameter values. When $d \approx m$, this can happen even with relatively small values of the trade-off parameter $c_{2}$.

a)

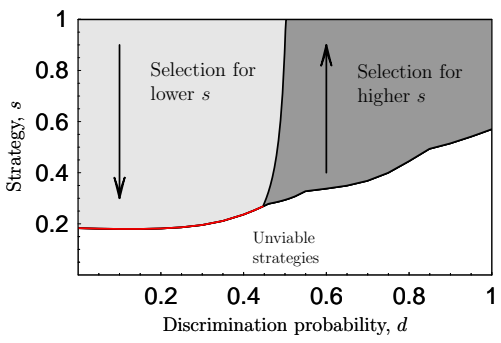

b)

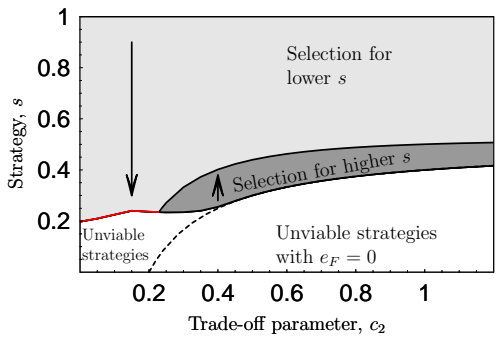

c)

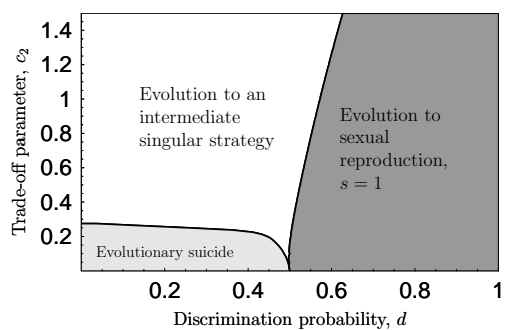

FiguRE 5. Dependence of the direction of selection and singular strategies as a function of a) the discrimination probability $d$ and b) the trade-off parameter $c_{2}$. c) Evolutionary scenarios as a function of $d$ and $c_{2}$. Parameters: $e_{F}=T\left(0.1+c_{2}(s-0.5)\right)$. a) $c_{2}=0.2$ b) $d=0.4$. Other parameters as in Figure 3a. 
In all observed cases, the intermediate evolutionarily singular strategy is both evolutionarily attracting and uninvadable (evolutionarily stable), so that it is the expected endpoint of evolution. This means that the evolving population is expected to remain monomorphic with respect to the evolving strategy $s$. It would have been especially interesting to observe evolutionary branching, in which the strategy of a monomorphic population first evolves to a singular strategy, and then the population becomes dimorphic, and disruptive selection causes the strategies of the two populations to diverge. Although we did not find such behaviour in the present model, we would like to point out that in a monomorphic population with an intermediate strategy $(0<s<1)$, both sexual and asexual behaviour does occur. Nevertheless, we can conclude that a metapopulation structure as such does not provide support for the evolutionary emergence, and evolutionarily stable coexistence of completely sexually and completely gynogenetically reproducing species.

\section{Discussion}

All offspring of a gynogenetically reproducing female are females, which may give it a reproductive advantage compared to sexually reproducing females, unless males are able to recognize gynogenetically reproducing females and refuse to mate with them. If the reproduction strategy is freely evolving in a large well-mixed population, strong discrimination of asexually reproducing females by males results in evolution to full sexuality, whereas mild discrimination leads to too small probability of sexual reproduction, so that the lack of males causes the extinction of the evolving population, resulting in evolutionary suicide $[12,17,18,38,42,43]$.

When the discrimination of asexually reproducing females is mild, the direct benefit of sexual reproduction to a female is smaller than the direct benefit of asexual reproduction. Sexual reproduction gives, however, indirect benefit, because the production of males increases the mating probability of all females in the future. Sexual reproduction can thus be considered as a cooperative act. Various mechanisms have been suggested to promote cooperation. Potential cooperation events may be repeated many times between the same individuals. If an individual decides not to cooperate, the partner is likely to remember such defecting act, and not to give help in the future either. Therefore, direct reciprocity may promote cooperation [50]. In case pairwise interactions are repeated, but not between the same individuals, by defecting one may get a bad reputation, and that way risk the possibility of receiving help in the future. Therefore, indirect reciprocity $[1,2,34-36,48]$ may promote cooperation, depending on the social norm [37]. These mechanisms are not likely to affect the evolution of sexual reproduction. Although reproduction results from a pairwise act of a male and a female, this is not a pairwise event in terms of cooperation. The benefit of sexually produced males is namely shared among all females in the population, and thus it can be considered as public-goods cooperation.

Assortment is a mechanism promoting cooperation [13], which applies also to public-goods cooperation. Whatever the mechanism causing assortment is, if a cooperating individual is more likely to be surrounded by cooperating individuals than a defecting individual is, the direct benefit received from them may promote cooperation. Assortment can also cause an individual to be surrounded by its relatives, and thus kin selection $[19,20]$ may promote cooperation. In a metapopulation structure assortment is naturally present. This gives motivation to study the evolution of sexual reproduction in a metapopulation model. A Levins-type metapopulation [24,25] model was investigated by [22], who assumed that an empty patch can be colonized by a sexually reproducing population, whereas an asexually reproducing population can only colonize patches already occupied by the sexually reproducing ones. By choosing suitable colonization and extinction rates, the sexually and asexually reproducing populations are able to coexist. These patch colonization rates, however, are not individual-level properties, and thus cannot be linked to the reproduction strategies of individuals. For this reason, we investigated here a structured metapopulation model, in which patch colonizations result from individual-level dispersal. With this kind of models, also the effect of the reproduction strategy to the local population dynamics can be incorporated in detail. Note, however, that the local populations in this article were assumed to be large, and therefore kin selection does not play a role here. We observed that the metapopulation structure 
as such does not alter the scenarios observed for the well-mixed population: strong discrimination again results in evolution to full sexuality, and mild discrimination leads to evolutionary suicide due to too small probability of sexual reproduction.

Based on the reasoning given above, in order to gynogenetically reproducing and sexually reproducing individuals to coexist, sexual reproduction should be accompanied with a property, which gives direct benefit to the sexually reproducing individual. Such direct benefit could result for example from differences in some other life-history trait. In a metapopulation context, dispersal behaviour is a natural choice. For this reason, we investigated the joint evolution of dispersal and the reproduction strategy. When these traits are allowed to evolve independently, for a given reproduction strategy there exists an evolutionarily singular dispersal strategy component, but again, mild discrimination leads to too low probability of sexual reproduction and evolutionary suicide, and strong discrimination to full sexual reproduction (Figure 3). Instead of independently evolving traits, imposing a trade-off between dispersal and the reproduction strategy can result in different evolutionary scenarios. We thus investigated a situation, in which the dispersal strategy of an individual is a function of its reproduction strategy. We observed that an intermediate evolutionarily singular reproduction strategy can emerge and be evolutionarily attracting, provided that the slope of the trade-off function is large enough. Such a situation thus enables the presence of sexual and asexual behaviour at the same time in this model, but it is due to females with a mixed reproduction trait. We did not observe disruptive selection resulting in the evolutionarily stable coexistence of fully sexual and fully asexual females. Even the ecological coexistence of fully sexual and fully asexual females does not appear to be possible. We thus conclude that a difference in the dispersal behaviour only seems not to be a sufficient mechanism to explain the observed coexistence of gynogenetic and sexual species.

What mechanisms remain that could explain the observed coexistence of gynogenetic and sexual species? First, there may be other differences than dispersal in the species. These differences, however, must be such that they provide an ecological niche for each reproductive trait $[28,47,52]$. Some other mechanisms are related to discrimination, including frequency-dependent male mate preference $[29,33]$. Considering the evolution of discrimination by males [23], it has been observed that for sperm-rich males, mating with a female is an act with very small cost. Refusing to mate with an asexually reproducing female thus provides a rather small benefit compared with the big cost of refusing to mate with a sexually reproducing one. Based on direct benefits and costs, discrimination is not likely to evolve. The situation is different in small populations in which kin selection matters [23]. In the model investigated in this article, discrimination is not expected to evolve. A metapopulation model with locally small populations $[31,41,45]$ would be a rather interesting setup to investigate the evolution of the reproduction strategy, as well as the evolution of discrimination. In such a model, producing males can be beneficial in the evolutionary sense, because the relatives of a sexually reproducing female benefit from such production of males. By a similar reasoning, mate discrimination by males may also evolve. We thus conclude that a mere metapopulation structure is not enough to support the coexistence of sexually and gynogenetically reproducing females, but there are metapopulation models in which such coexistence is plausible. We, however, leave such investigation for future work.

\section{Appendix: Metapopulation reproduction ratio of the mutant}

Assume, that the metapopulation is at a population-dynamical equilibrium, in which $I^{M}$ and $I^{i}, i=$ $1, \ldots, n$ are the amounts of immigrants for males and females. As explained in the main text, all patches of age $\tau$ have the same population sizes $M_{\tau}, F_{\tau}^{1}, \ldots, F_{\tau}^{n}$, which can be solved from (3.7) with $M_{0}=F_{0}^{1}=$ $\cdots=F_{0}^{n}=0$. Now we investigate what is expected to happen to a mutant individual with strategy $s_{\text {mut }}$ in the environment set by these residents.

Consider a small immigrating mutant population of size $F_{0}^{\text {mut }}$ which arrives in a patch. The probability that this patch has age $\tau$ is $v(\tau)$. Because catastrophes happen after immigration, this mutant population is present in that patch in the beginning of the next season with probability $1-\mu$. At that time, this patch has age $\tau+1$ with probability $(1-\mu) v(\tau)=v(\tau+1)$. At that time the mutant population size 
is $F_{0}^{\text {mut }}$, and the quantity $F^{\text {mut }}(\tau+1, t)$ denotes the mutant population size $t$ time steps later. Because the mutant population is rare, no new mutant immigrants are expected to arrive in the patch. If no catastrophes will happen, the local mutant population will thus grow according to

$$
\left\{\begin{aligned}
F^{\mathrm{mut}}(\tau+1,0)= & F_{0}^{\text {mut }} \\
F^{\mathrm{mut}}(\tau+1, t+1)= & \left(1-e_{F, \text { mut }}\right) f\left(N_{\tau+1+t}\right) b\left(\hat{F}_{\tau+1+t}, M_{\tau+1+t}\right) \\
& {\left[\left(1-s_{\mathrm{mut}}\right)(1-d)+s_{\text {mut }}(1-m)\right] F^{\text {mut }}(\tau+1, t) . }
\end{aligned}\right.
$$

The per capita number of emigrants that this newly founded mutant colony is expected to produce during its entire lifetime is equal to

$$
\begin{aligned}
E(\tau+1)=\frac{1}{F_{0}^{\text {mut }}} \sum_{t=0}^{\infty} e_{F, \text { mut }} f\left(N_{\tau+1+t}\right) b\left(\hat{F}_{\tau+1+t}, M_{\tau+1+t}\right) \\
{\left[\left(1-s_{\text {mut }}\right)(1-d)+s_{\text {mut }}(1-m)\right] F^{\text {mut }}(\tau+1, t)(1-\mu)^{t}, }
\end{aligned}
$$

where $(1-\mu)^{t}$ is the probability that a catastrophe has not happened in $t$ time steps.

Finally, the expected number of surviving mutant emigrants produced by a mutant who arrives in a patch is

$$
R\left(s_{\mathrm{mut}}, E_{\mathrm{res}}\right)=\pi \sum_{\tau=0}^{\infty} v(\tau+1) E(\tau+1) .
$$

For an efficient algorithm for the calculation of this quantity, see [39].

Acknowledgements. The author highly appreciates the constructive feedback that was given by anonymous reviewers. The author also wishes to thank the Academy of Finland for financial support (project number 128323)

\section{References}

[1] R. D. Alexander. Darwinism and human affairs. Seattle, University of Washington Press, 1979.

[2] R. D. Alexander. The biology of moral systems. New York, Aldine de Gruyter, 1987.

[3] L. W. Beukeboom, R. C. Vrijenhoek. Evolutionary genetics and ecology of sperm-dependent parthenogenesis. J. Evol. Biol, 11 (1998), 755-782.

[4] Å. Brännström, U. Dieckmann. Evolutionary dynamics of altruism and cheating among social amoebas. Proc. R. Soc. London B, 272 (2005), 1609-1616.

[5] Å. Brännström, D. J. T. Sumpter. The role of competition and clustering in population dynamics. Proc. R. Soc. London B, 272 (2005), 2065-2072.

[6] F. B. Christiansen. On conditions for evolutionary stability for a continuously varying character. Am. Nat., 138 (1991), 37-50.

[7] M. Doebeli, C. Hauert, T. Killingback. The evolutionary origin of cooperators and defectors. Science, 306 (2004), 859-862.

[8] I. Eshel. Evolutionary and continuous stability. J. Theor. Biol., 103 (1983), 99-111.

[9] H. Eskola, S. A. H. Geritz. On the mechanistic derivation of various discrete-time population models. Bull. Math. Biol., 69 (2007), 329-346.

[10] H. Eskola, K. Parvinen. On the mechanistic underpinning of discrete-time population models with Allee effect. Theor. Popul. Biol., 72 (2007), 41-51.

[11] H. Eskola, K. Parvinen. The Allee effect in mechanistic models based on inter-individual interaction processes. Bull. Math. Biol., 72 (2010), 184-207.

[12] R. Ferrière. Adaptive responses to environmental threats: evolutionary suicide, insurance, and rescue. Options Spring 2000, IIASA, Laxenburg, Austria, 12-16, 2000.

[13] J. A. Fletcher, M. Doebeli. A simple and general explanation for the evolution of altruism. Proc. R. Soc. London B, 276 (2009), 13-19.

[14] S. A. H. Geritz, É. Kisdi, G. Meszéna, J. A. J. Metz. Evolutionarily singular strategies and the adaptive growth and branching of the evolutionary tree. Evol. Ecol., 12 (1998), 35-57.

[15] S. A. H. Geritz, J. A. J. Metz, É. Kisdi, G. Meszéna. Dynamics of adaptation and evolutionary branching. Phys. Rev. Lett., 78 (1997), 2024-2027.

[16] M. Gyllenberg, J. A. J. Metz. On fitness in structured metapopulations. J. Math. Biol., 43 (2001), 545-560. 
[17] M. Gyllenberg, K. Parvinen. Necessary and sufficient conditions for evolutionary suicide. Bull. Math. Biol., 63 (2001), 981-993.

[18] M. Gyllenberg, K. Parvinen, U. Dieckmann. Evolutionary suicide and evolution of dispersal in structured metapopulations. J. Math. Biol., 45 (2002), 79-105.

[19] W. D. Hamilton. The genetical evolution of social behaviour I. J. Theor. Biol, 7 (1964), 1-16.

[20] W. D. Hamilton. The genetical evolution of social behaviour II. J. Theor. Biol, 7 (1964), 17-52.

[21] K. Heubel, D. Rankin, H. Kokko. How to go extinct by mating too much: population consequences of male mate choice and efficiency in a sexual-asexual species complex. Oikos, 118 (2009), 513-520.

[22] H. Kokko, K. Heubel, D. Rankin. How populations persist when asexuality requires sex: the spatial dynamics of coping with sperm parasites. Proc. R. Soc. London B, 275 (2008), 817-825.

[23] H. Kokko, K. U. Heubel. Prudent males, group adaptation, and the tragedy of the commons. Oikos, 120 (2011), $641-656$.

[24] R. Levins. Some demographic and genetic consequenses of environmental heterogeneity for biological control. Bull. Entomol. Soc. Am., 15 (1969), 237-240.

[25] R. Levins. Extinction. In M. Gerstenhaber, editor, Some Mathematical Problems in Biology. American Mathematical Society, Providence, RI, (1970), 77-107.

[26] H. Matsuda. Evolutionarily stable strategies for predator switching. J. Theor. Biol, 115 (1985), 351-366.

[27] J. Maynard Smith. Evolution and the theory of games. Amer. Sci., 64 (1976), 41-45.

[28] J. A. Mee, F. Noddin, J. R. Hanisch, W. M. Tonn, C. A. Paszkowski. Diets of sexual and sperm-dependent asexual dace (Chrosomus spp.): relevance to niche differentiation and mate choice hypotheses for coexistence. Oikos, 122 (2013), 998-1008.

[29] J. A. Mee, S. P. Otto. Variation in the strength of male mate choice allows long-term. Evolution, 64 (2010), $2808-2819$.

[30] J. A. J. Metz, S. A. H. Geritz, G. Meszéna, F. J. A. Jacobs, J. S. van Heerwaarden. Adaptive dynamics, a geometrical study of the consequenses of nearly faithful reproduction. In S. J. van Strien and S. M. Verduyn Lunel, editors, Stochastic and Spatial Structures of Dynamical Systems. North-Holland, Amsterdam, (1996), 183-231.

[31] J. A. J. Metz, M. Gyllenberg. How should we define fitness in structured metapopulation models? Including an application to the calculation of ES dispersal strategies. Proc. R. Soc. London B, 268 (2001), 499-508.

[32] J. A. J. Metz, R. M. Nisbet, S. A. H. Geritz. How should we define "fitness" for general ecological scenarios? Trends Ecol. Evol., 7 (1992), 198-202.

[33] W. S. Moore. Components of fitness in a unisexual fish Poeciliopsis monacha-occidentalis. Evolution, 30 (1976), 564578.

[34] M. A. Nowak, K. Sigmund. The dynamics of indirect reciprocity. J. Theor. Biol, 194 (1998), 561-574.

[35] M. A. Nowak, K. Sigmund. Evolution of indirect reciprocity by image scoring. Nature, 393 (1998), 573-577.

[36] M. A. Nowak, K. Sigmund. Evolution of indirect reciprocity. Nature, 437 (2005), 1291-1298.

[37] H. Ohtsuki, Y. Iwasa. The leading eight: Social norms that can maintain cooperation by indirect reciprocity. J. Theor. Biol., 239 (2006), 435-444.

[38] K. Parvinen. Evolutionary suicide. Acta Biotheoretica, 53 (2005), 241-264.

[39] K. Parvinen. Evolution of dispersal in a structured metapopulation model in discrete time. Bull. Math. Biol., 68 (2006), 655-678.

[40] K. Parvinen. Evolutionary suicide in a discrete-time metapopulation model. Evol. Ecol. Res., 9 (2007), 619-633.

[41] K. Parvinen. Adaptive dynamics of altruistic cooperation in a metapopulation: Evolutionary emergence of cooperators and defectors or evolutionary suicide? Bull. Math. Biol., 73 (2011), 2605-2626.

[42] K. Parvinen, U. Dieckmann. Self-extinction through optimizing selection. J. Theor. Biol, 333 (2013), 1-9.

[43] K. Parvinen, U. Dieckmann. Evolutionary suicide. In U. Dieckmann and J. A. J. Metz, editors, Elements of Adaptive Dynamics. Cambridge University Press, (in press).

[44] K. Parvinen, U. Dieckmann, M. Gyllenberg, J. A. J. Metz. Evolution of dispersal in metapopulations with local density dependence and demographic stochasticity. J. Evol. Biol, 16 (2003), 143-153.

[45] K. Parvinen, J. A. J. Metz. A novel fitness proxy in structured locally finite metapopulations with diploid genetics, with an application to dispersal evolution. Theor. Popul. Biol., 73 (2008), 517-528.

[46] C. Rueffler, M. Egas, J. A. J. Metz. Evolutionary predictions should be based on individual-level traits. Am. Nat., 168 (2006), E148-E162.

[47] D. Schley, C. P. Doncaster, T. Sluckin. Population models of sperm-dependent parthenogenesis. J. Theor. Biol, 229 (2004), 559-572.

[48] R. Sugden. The Evolution of Rights, Co-operation and Welfare. Oxford: Blackwell, 1986.

[49] D. J. T. Sumpter, D. S. Broomhead. Relating individual behaviour to population dynamics. Proc. R. Soc. London B, 268 (2001), 925-932.

[50] R. Trivers. The Evolution of Reciprocal Altruism. Q Rev Biol, 46 (1971), 35-57.

[51] P. H. Van Tienderen, G. De Jong. Sex ratio under the haystack model: Polymorphism may occur. J. Theor. Biol., 122 (1986), 69-81.

[52] R. C. Vrijenhoek. Factors affecting clonal diversity and coexistence. Am. Zool., 19 (1979), 787-797. 